\title{
Violência doméstica à mulher: percepção e abordagem profissional na atenção básica na pandemia de Covid-19*
}

Violence against women: perception and professional approach in primary health care during the Covid-19 pandemic

Violencia intrafamiliar contra la mujer: percepción y enfoque profesional en atención primaria en la pandemia Covid-19

\section{Bruna Odorcik ${ }^{\mathrm{I}}$, Brígida da Penha Ferraz ${ }^{\mathrm{II}}$, Karina Castilhos Bastos ${ }^{\mathrm{III}}$, Maíra Rossetto ${ }^{\mathrm{IV}}$}

Resumo: Objetivo: analisar a abordagem de profissionais de saúde na identificação da violência doméstica às mulheres e a sua percepção sobre os casos durante a pandemia da Covid-19 em Centros de Saúde da Família. Método: pesquisa qualitativa realizada em duas unidades de saúde, mediante entrevistas semiestruturadas, com 23 profissionais de saúde. A coleta de dados ocorreu entre novembro de 2019 e fevereiro de 2021, e os dados foram organizados por meio da análise de conteúdo. Resultados: as categorias mostraram que os profissionais sabem identificar os tipos de violência doméstica, mas que necessitam de maior sensibilização para acolher, identificar e notificar casos. Na percepção dos profissionais durante a pandemia, ocorreu um aumento da violência atribuído ao isolamento social. Conclusão: necessita-se melhorar o trabalho em rede, aumentar as qualificações dos profissionais, oferecendo técnicas de acolhimento e escuta qualificada às mulheres que passam por situações de violência doméstica.

Descritores: Violência Contra a Mulher; Atenção Primária à Saúde; Profissionais da Saúde; Covid-19; Notificação de Abuso

Abstract: Objective: To analyze the approach of health professionals in the identification of violence against women and their perception of the cases during the Covid-19 pandemic in Family Health Centers. Method: Qualitative study carried out in two health units, through semi-structured interviews with 23 health professionals. Data were collected between November 2019 and February 2021, and organized with the use of content analysis. Results: the categories created showed that health professionals know how to identify the types of domestic violence, but need greater awareness to support, identify and report the cases. According to the perception of the health professionals during the

\footnotetext{
I Psicóloga, Especialista em Saúde coletiva. Universidade do Oeste de Santa Catarina. Chapecó, Santa Catarina, Brasil. E-mail: bruna.odorcik@hotmail.com, Orcid: https://orcid.org/0000-0002-0876-0843

II Acadêmica do curso de Medicina da Universidade Federal da Fronteira Sul. Chapecó, Santa Catarina, Brasil. E-mail: brigidaferraz00@gmail.com, Orcid: https://orcid.org/0000-0003-0288-8763

III Acadêmica do curso de Medicina da Universidade Federal da Fronteira Sul. Chapecó, Santa Catarina, Brasil. E-mail: karinacastilhosbastos@gmail.com, Orcid: https://orcid.org/0000-0002-8439-7230.

IV Enfermeira. Professora de Saúde coletiva do curso de Medicina pela Universidade Federal da Fronteira Sul - Campus Chapecó. Chapecó, Santa Catarina, Brasil. E-mail: maira.rossetto@uffs.edu.br, Orcid: https://orcid.org/0000-0002-5683-4835

* Extraído do Trabalho de Conclusão de Curso do programa de pós-graduação em Saúde Coletiva na Universidade Federal da Fronteira Sul, ano de 2021.
} 
pandemic, there was an increase in violence attributed to social distancing. Conclusion: it is necessary to improve networking, provide better training to health professionals, offering support and active listening skills to women who face situations of domestic violence.

Descriptors: Violence Against Women; Primary Health Care; Health Professionals; Covid-19; Mandatory reporting

Resumen: Objetivo: analizar el abordaje de los profesionales de la salud en la identificación de la violencia intrafamiliar contra la mujer y su percepción de los casos durante la pandemia Covid-19 en los Centros de Salud de la Familia. Método: investigación cualitativa realizada en dos unidades de salud, mediante entrevistas semiestructuradas con 23 profesionales de la salud. La recolección de datos se llevó a cabo entre noviembre de 2019 y febrero de 2021, y los datos se organizaron mediante análisis de contenido. Resultados: las categorías mostraron que los profesionales saben identificar los tipos de violencia intrafamiliar, pero necesitan una mayor conciencia para recibir, identificar y notificar los casos. En la percepción de los profesionales durante la pandemia, hubo un aumento de la violencia atribuida al aislamiento social. Conclusión: existe la necesidad de mejorar el networking, incrementar la calificación de los profesionales, ofreciendo técnicas de recepción y escucha calificada a las mujeres que viven situaciones de violencia intrafamiliar.

Descriptores: Violencia Contra la Mujer; Atención Primaria de Salud; Personal de Salud; Covid-19; Notificación Obligatoria

\section{Introdução}

A atenção básica é definida como porta de entrada dos serviços de saúde pela Política Nacional de Atenção Básica (Pnab) e pode atuar de forma significativa no acolhimento das mulheres vítimas de violência doméstica. ${ }^{1-2}$ Este agravo pode ser conceituado como qualquer ato violento ligado ao gênero que resulte, ou possa resultar, em dano ou sofrimento físico, sexual ou mental para as mulheres, incluindo ameaças de prática de tais atos e a coerção ou privação arbitrária da liberdade, seja em vida pública ou privada. ${ }^{3}$

No Brasil, a aplicação da Lei Maria da Penha, consiste em estratégias para prevenir, punir e mitigar a violência contra as mulheres revelada de diferentes formas. ${ }^{4}$ Entretanto, apesar da lei, os números relacionados a esse agravo têm piorado nos últimos anos. Esse cenário pode ser observado ao se levarem em conta dados do Conselho Nacional de Justiça, que estimou a presença de cerca de 1 milhão de processos referentes a violência doméstica contra mulher no país. ${ }^{5}$ Em relação ao Estado de Santa Catarina, esse aumento pode ser pontuado conforme dados do Sistema Integrado de Segurança Pública, que demonstram um crescimento de $83 \%$ dos feminicídios entre janeiro e março de 2019, em comparação ao mesmo período do ano anterior. ${ }^{6}$ 
Com a ocorrência da pandemia do coronavirus disease (Covid-19), os indicadores ganharam destaque nacional, com agravamento do cenário de violência. Tal fato foi demonstrado pelos dados do Anuário Brasileiro de Segurança Pública, que, ao comparar os anos de 2019 e 2020, identificou a ocorrência de 1.350 feminicídios no ano de 2020, com aumento de $0,7 \%$ se comparado ao ano anterior; 694.131 ligações de violência doméstica no 190, com acréscimo de 16,3\%; e 294.440 medidas protetivas de urgência concedidas pelo tribunal de justiça, passando para 3,6\%. A Organização das Nações Unidas (ONU) Mulheres Brasil destaca que a pandemia tem piorado condições de vida e de trabalho e agravado os casos de violência sexual e de gênero contra mulheres e meninas. ${ }^{8}$

O recrudescimento dos casos de violência doméstica durante a pandemia tem sido atribuído ao isolamento social e pensado por meio de uma análise interseccional entre as categorias desigualdades raciais, de gênero e de classe social, oriundas das relações opressivas do sistema patriarcal. ${ }^{9}$ Mas as situações apresentadas não são novas, o que ocorre é a exacerbação de problemas, reforçados por modelos de pensamentos retrógrados e misóginos sobre o que é ser mulher na sociedade brasileira. ${ }^{10}$

Os números permitem pontuar que este agravo é um problema de saúde pública e, dessa forma, os profissionais da saúde podem ser fundamentais na promoção e na execução de práticas que possam identificar e combater a violência doméstica. Nesse sentido, torna-se necessário conhecer a abordagem de profissionais de saúde na atenção básica diante da identificação de casos de violência doméstica contra mulheres, considerando especialmente o período pandêmico.

As atitudes desejáveis por parte de profissionais têm mostrado que as mulheres tendem a estimar a escuta qualificada, a não culpabilização e o oferecimento das possibilidades de atendimento na rede de serviços. ${ }^{2,11}$ Neste sentido, os profissionais que acolhem precisam deste olhar para as necessidades singulares de cada mulher, ofertando serviços da rede intersetorial e cuidados à saúde mental. ${ }^{12}$ Outra importante ação dos profissionais relaciona-se com a notificação dos casos de violência doméstica, que durante a pandemia podem ter sido ainda maiores, pois as mulheres estavam isoladas e com mais dificuldade de chegar aos serviços de saúde. ${ }^{9}$ 
Violência doméstica à mulher: percepção e abordagem profissional na aten... | 4

Para tanto, formulou-se, como objetivo, analisar a abordagem de profissionais de saúde na identificação da violência doméstica às mulheres e a sua percepção sobre os casos durante a pandemia da Covid-19 em Centros de Saúde da Família (CSF).

\section{Método}

Trata-se de uma pesquisa de abordagem qualitativa, do tipo exploratória, realizada em dois CSF na cidade de Chapecó, Santa Catarina, Brasil. Os CSF foram escolhidos por possuírem distintas condições, sendo um situado em áreas de maior vulnerabilidade e o outro cuja área adscrita são bairros com melhores índices de desenvolvimento social. Os dois bairros foram indicados pela secretaria municipal de saúde como cenários para coleta de dados, sendo locais na cidade com ocorrência elevada de casos de violência doméstica.

Os critérios de inclusão dos participantes da pesquisa foram ser profissional da saúde e estar disponível para agendar entrevista no período da coleta de dados (não estar em férias ou licença). Já o critério de exclusão foi não atender diretamente aos usuários nos CSF. As pesquisadoras não conheciam as unidades de saúde nem os profissionais vinculados aos serviços, sendo que os contatos foram realizados por meio da secretaria municipal de saúde.

A coleta de dados ocorreu em duas etapas. No primeiro momento, em novembro de 2019, de modo presencial, os dados foram coletados pela pesquisadora por meio de entrevistas. Anteriormente, houve contato com a coordenação de cada unidade de saúde e identificados os possíveis participantes do estudo, sendo feita uma lista de profissionais atuantes nos CSF. Em seguida, foram agendados dias e horários para a entrevista em sala disponível na unidade de saúde e de acordo com o melhor horário para o profissional. Dos profissionais agendados presencialmente, não ocorreram recusas na participação. Após o aceite do profissional para participar da pesquisa, os participantes assinaram o Termo de Consentimento Livre e Esclarecido (TCLE). As entrevistas foram realizadas pela mesma pesquisadora, com experiências anteriores na aplicação de entrevistas. 
5 | Odorcik B, Ferraz BP, Bastos KC, Rossetto M

As questões foram aplicadas aos profissionais por meio de uma entrevista que durou aproximadamente 40 minutos. Estas foram gravadas e armazenadas em gravador de voz portátil, sendo posteriormente transcritas pela pesquisadora na íntegra, com a substituição do nome dos profissionais entrevistados por códigos. A pesquisadora que realizou a coleta dos dados é psicóloga em serviço privado e não tinha vínculos com os profissionais dos serviços pesquisados.

A segunda etapa ocorreu de modo on-line, por meio de questionário, devido ao início da pandemia da Covid-19, com a impossibilidade de coleta presencial. Foi submetida uma emenda ao comitê de ética em pesquisa, alterando o modo de coleta, as questões relacionadas aos riscos, benefícios, aplicação de TCLE e questionário, com o acréscimo de uma pergunta referente à pandemia (respondida por 15 pessoas). Após a aprovação da emenda, a coleta de dados on-line ocorreu entre dezembro de 2020 e fevereiro de 2021. O questionário e o TCLE foram enviados por meio de link eletrônico às coordenadoras dos CSF pela secretaria municipal de saúde, que fizeram o reenvio para os profissionais de saúde por meio do aplicativo de celular.

O instrumento de coleta dos dados abordava questões referentes à identificação pessoal e profissional; entendimento do que é violência doméstica; experiência profissional quanto a suspeita, identificação e conduta; conhecimento acerca da rede de atenção a pessoas vítimas de violência doméstica do município; entendimento quanto ao suporte que deverá ser ofertado à vítima, como também sobre a identificação da necessidade de capacitação para os profissionais, além da percepção quanto ao aumento dos casos no cenário da pandemia (aplicada apenas no questionário on-line após o início da pandemia). As entrevistas gravadas na primeira fase foram transcritas e não ocorreu devolução aos participantes.

O tamanho amostral não foi definido a priori, todos os profissionais das duas unidades de saúde foram convidados a participar do estudo, sendo que, de um total de 34 participantes, 23 responderam ao instrumento de coleta de dados. No envio on-line do instrumento de coleta de dados, alguns profissionais não responderam ao questionário, não sendo possível mensurar os motivos da recusa na participação. No total, 23 profissionais participaram da pesquisa, sendo oito 
Violência doméstica à mulher: percepção e abordagem profissional na aten... | 6

na etapa presencial e 15 na etapa on-line

Foi desenvolvida análise de conteúdo. ${ }^{13} \mathrm{Na}$ pré-análise, após reunir em um único documento todas as entrevistas transcritas na íntegra (primeira fase da coleta) e as respostas obtidas pelos questionários eletrônicos (segunda fase da coleta), foi realizada a leitura flutuante do material. Além disso, realizou-se também a organização dos dados coletados, selecionando o que havia de relevante para ser trabalhado, de modo a permitir a identificação das ideias que continham sentido para serem analisadas nas etapas seguintes. Ressalta-se que, para a seleção dos dados, foram cumpridas as regras da exaustividade, homogeneidade e pertinência. ${ }^{13}$

$\mathrm{Na}$ exploração do material, procurou-se verificar se as ações da fase anterior foram devidamente concluídas, para assim proceder com a codificação e decomposição das construções coletadas. Para a codificação, foram estabelecidas as unidades de registro por temas, e recortadas e agrupadas as falas de cada tema. Foram criadas as categorias que transformam os dados brutos em dados organizados, fornecendo uma representação simplificada. ${ }^{13}$ Em cada categoria, foram reunidas as unidades de registros que apresentavam relação e operou-se uma nomeação, por meio de um título abrangente, à categoria, a exemplo: Percepção do profissional: identificação do que é violência doméstica e como se manifesta; Necessidade de orientação externa: possibilidade de projeto para orientação da equipe; Opinião do profissional quanto à relevância do tema: a discussão faz sentido nos CSF de Chapecó?; Aumento dos casos de violência doméstica: contexto Covid-19, existiu aumento devido ao isolamento? Ainda na fase de exploração, foi realizada a enumeração das unidades de registro por meio da contagem da frequência com que essas unidades apareceram nas falas dentro de cada categoria, considerando que a importância de cada unidade de registro é diretamente proporcional ao número de aparições. Também foi utilizada a enumeração por direção, com os polos direcionais “conhece/não conhece/conhece pouco” em uma determinada categoria.

Por último, na fase de tratamento, inferências e interpretações dos resultados, foram empreendidos esforços para que os resultados obtidos fossem significativos e válidos. Nesta etapa se "permitem estabelecer quadros de resultados, diagramas, figuras e modelos, os quais condensam 
e põem em relevo as informações fornecidas pela análise". ${ }^{13: 127}$ A forma de inferência utilizada foi a específica, pois buscou-se responder ao problema de pesquisa. Para isso, foram construídos quadros que permitiram a visualização facilitada de informações, como também possibilitaram constantes retornos que auxiliaram na interpretação dos resultados.

O projeto de pesquisa foi submetido ao Comitê de Ética e Pesquisa da Universidade Federal da Fronteira Sul e aprovado sob o parecer n 3.687.958, em 6 de novembro de 2019. A emenda foi aprovada pelo parecer $n^{\circ}$ 4.301.333, em 26 de setembro de 2020. A pesquisa foi conduzida de acordo com os padrões éticos (Resoluções 466/2012- 510/2016 - 580/2018, do Ministério da Saúde).

\section{Resultados}

No total, 23 profissionais participaram da pesquisa, sendo oito na etapa presencial e 15 na etapa on-line. No Quadro 1, estão apresentadas algumas características dos participantes da pesquisa, como a profissão, a idade, o ano de formação, a experiência profissional anterior e a área de atuação no CSF/tempo de atuação.

Quadro 1 - Características dos participantes da pesquisa

\begin{tabular}{|c|c|c|c|c|c|}
\hline Nome & Profissão & Idade & $\begin{array}{l}\text { Ano de } \\
\text { formação }\end{array}$ & Experiência profissional anterior & $\begin{array}{l}\text { Cargo / tempo de } \\
\text { atuação }\end{array}$ \\
\hline $\mathrm{P} 1$ & Enfermeira & 45 & 1997 & Docente & $\begin{array}{l}\text { Coordenadora } \\
23 \text { anos }\end{array}$ \\
\hline $\mathrm{P} 2$ & Enfermeira & 37 & 2006 & Apenas acadêmica & 13 anos \\
\hline P3 & Enfermeira & 30 & 2010 & Hospitalar & 09 meses \\
\hline $\mathrm{P} 4$ & Enfermeira & 33 & 2009 & Não possui & 10 anos \\
\hline P5 & Enfermeira & 27 & 2015 & $\begin{array}{c}\text { Hospital, Unidade de Pronto } \\
\text { Atendimento (UPA) }\end{array}$ & $\begin{array}{l}\text { Coordenadora } \\
04 \text { anos }\end{array}$ \\
\hline P6 & Enfermeiro & 44 & 2007 & UPA & 06 anos \\
\hline P7 & Médica & 34 & 2014 & Preceptoria & 5 anos \\
\hline P8 & Médica & 33 & 2015 & UPA, Pronto Atendimento, Samu & 01 ano \\
\hline
\end{tabular}


Violência doméstica à mulher: percepção e abordagem profissional na aten... $\mid 8$

\begin{tabular}{|c|c|c|c|c|c|}
\hline P9 & Dentista & 37 & 2004 & Dentista no setor privado & 14 anos \\
\hline P10 & Dentista & 41 & 2002 & Não possui & 17 anos \\
\hline P11 & Assistente social & 35 & 2010 & Agente comunitário de saúde (ACS) & 06 anos \\
\hline P12 & Psicóloga & 54 & 2003 & Psicóloga & 11 anos \\
\hline P13 & Farmacêutica & 39 & 2006 & Hospital, farmácias privadas & 09 anos \\
\hline P14 & $\begin{array}{c}\text { Auxiliar de saúde } \\
\text { bucal (ASB) }\end{array}$ & 42 & Não recorda & $\begin{array}{l}\text { Atendente de farmácia, } \\
\text { recepcionista, telefonista }\end{array}$ & 14 anos \\
\hline P15 & $\begin{array}{l}\text { Auxiliar de } \\
\text { enfermagem }\end{array}$ & 35 & 2008 & Não possui & 10 anos \\
\hline P16 & $\begin{array}{l}\text { Auxiliar de } \\
\text { enfermagem }\end{array}$ & 45 & 2012 & Não possui & 10 anos \\
\hline P17 & $\begin{array}{c}\text { Auxiliar } \\
\text { administrativo }\end{array}$ & 38 & 2011 & Unimed, Sadia, Unochapecó & 20 dias \\
\hline P18 & ACS & 53 & Não recorda & Vendedora, costureira & 14 anos \\
\hline P 19 & ACS & 30 & Não recorda & Vendedora, agente de endemias & 03 anos \\
\hline P20 & ACS & 27 & 2018 & Comércio & 02 anos \\
\hline P21 & ACS & 38 & 2011 & Não possui & 10 anos \\
\hline $\mathrm{P} 22$ & ACS & 40 & 2009 & Frigorífico & 02 anos e 06 meses \\
\hline $\mathrm{P} 23$ & ACS & 35 & 2000 & Não possui & 01 ano \\
\hline
\end{tabular}

O perfil dos participantes foi composto por profissionais do sexo feminino, sendo que a maioria eram enfermeiras ou ACS, com idade entre 30 e 39 anos, formados entre 2000 e 2010, com trabalhos anteriores à atual ocupação na área da saúde e na atual função há mais de 10 anos.

A partir da análise dos dados, são apresentadas quatro categorias: Identificação do que é violência doméstica: [...] são várias formas de violência, não só física [...]; Necessidade de orientação para a equipe: [...] capacitação para conhecer a rede de serviços [...], [...] identificar casos suspeitos e direcionar o atendimento [...]; Discutir violência é importante: [...] falta sensibilizar ainda mais os profissionais, e Contexto da pandemia da Covid-19: [...] aumentou [a violência] pela situação de 
estresse e maior tempo juntos em casa.

Identificação do que é violência doméstica: [...] são várias formas de violência, não só física [...]

Entre esses profissionais, há um reconhecimento do que pode ser definido como violência. Ainda, a privação de patrimônio, agressão moral e sexual foram citadas, mas com menor frequência. Os depoimentos apontaram como os participantes identificam a violência doméstica em seu cotidiano de trabalho.

Então, são várias formas de violência, não só física [...] tem muitas mulheres que são violentadas psicologicamente, financeiramente, muitas vezes por bullying [...] E acaba talvez não se dando conta que estão sendo agredidas verbalmente[...]. (P1)

Tanto verbal quanto física, os pacientes vêm e contam o que elas passam em casa, às vezes ser diminuída, pelo simples fato de ser mulher ou a violência de bater mesmo. (P7)

Toda violência psicológica, verbal, física que as pessoas [...] ameaças, que as pessoas fazem para outra pessoa [...] até a questão de você inibir a pessoa, usar algum, algum problema que ela tem físico, para inibir ela de alguma forma [...] (P2)

[...] todo tipo de violência tanto física quanto verbal, que ocorre na residência, tanto a nível familiar, não só com vínculos de sangue, mas de convivio [...] tanto com criança, homem, mulher [...]. (P3)

Qualquer ato que viole a integridade física ou psicológica de uma pessoa, ocasionada dentro do lar ou por familiares/companheiros/as. (P9)

Toda e qualquer violação, opressão, ameaça, bater, xingar, que venha ocorrer contra a mulher no seu ambiente familiar em uma relação afetiva. (P11)

O conceito de violência doméstica está apreendido e relatado nos depoimentos dos participantes, pois os profissionais identificam o que é violência e suas diferentes formas de ocorrência nas usuárias atendidas por eles na atenção básica. Eles conseguem perceber as diferentes manifestações da violência física, verbal, psicológica, moral e financeira nas mulheres que atendem.

Necessidade de orientação para a equipe: [...] capacitação para conhecer a rede de serviços [...], [...] identificar casos suspeitos e direcionar o atendimento [...]

Os profissionais reconhecem a importância de momentos de orientação da equipe para atuar na assistência a mulheres em situação de violência. Quanto à necessidade da implementação de capacitações para a equipe de saúde sobre identificação e abordagem de mulheres vítimas de 
Violência doméstica à mulher: percepção e abordagem profissional na aten... | 10 violência doméstica, os participantes perceberam a necessidade de orientações para a realização adequada da identificação, do cuidado e do direcionamento das vítimas. Os processos formativos também são necessários principalmente para desenvolver a confiança para dar uma orientação de forma assertiva, quando necessário, conforme os depoimentos dos participantes.

Capacitação para conhecer a rede de serviços, identificar casos suspeitos e direcionar o atendimento. (P13)

Creio que seja necessário capacitações sobre o assunto para os profissionais da saúde. Não me sinto confortável em orientar, encaminhar ou sugerir açôes para pacientes vítimas ou que eu tenha suspeitado serem vítimas de violência. Seja por desconhecimento ou mesmo por receio de estar "invadindo" a privacidade da/o paciente. (P9)

Capacitação para alguns profissionais compreenderem que mulheres retornam para os agressores por inúmeras razóes psicológicas e não por gostarem de apanhar. (P12)

Ainda, a formação adequada poderia romper com julgamentos de alguns profissionais, preparando-os para o cuidado.

Capacitação em primeiro lugar, para que os profissionais possam romper com os julgamentos, enfim o que está velado por trás de muitas condutas profissionais. Melhorar o atendimento e capacitar para que todos possam realizar um acolhimento e identificar alguma situação de violência, capacitar sobre a rede de apoio e o protocolo de atendimento às vítimas de violência sexual, reforçar sobre a notificação compulsória. Criar estratégias junto à comunidade para que essa também denuncie situaçôes de violência que ocorrem na casa ao lado. Discutir dados epidemiológicos para pensar ações educativas no território. (P11)

Na formação isso deve ser abordado, pois será uma realidade cada vez mais nos serviços de saúde. (P13)

Os profissionais também demonstram a necessidade de melhorar a notificação nos registros de violência para produção de dados mais confiáveis sobre este agravo.

A gente sabe que acontece muito, mesmo os dados estatísticos são subnotificados, mas a realidade a gente não sabe [...] é um tema importante que tem que ser debatido, que acontece muito. (P10)

Na maioria das vezes foi relato da mulher sobre violência cometida há poucas horas com o companheiro, sendo necessário notificar e preencher questões burocráticas. $(\mathrm{P} 17)$

Os profissionais apontam que a equipe de saúde precisa ser orientada para, assim, se sentir mais segura no momento do encaminhamento das mulheres na rede de atenção. São apontadas a formação e as capacitações permanentes como uma forma de melhorar o conhecimento e a capacidade de notificação e geração de dados sobre este agravo. 
Discutir violência é importante: [...] falta sensibilizar ainda mais os profissionais

Quando questionados sobre a assistência às mulheres, as respostas dos profissionais demonstram a importância da discussão da temática, pois consideraram ser necessária uma sensibilização dos profissionais para o cuidado das mulheres vítimas de violência.

[...] pode ser outro serviço que tenha casa, abrigo das mulheres, tem vários serviços que podem ser a porta de entrada, que também fazem parte da rede de atenção à saúde da mulher. (P1)

[...] acredito que deveria ter uma rede que auxiliasse meio que junto até, com a delegacia com a mulher, uma coisa que pudesse ter um atendimento não só psicológico, mas médico também. (P3)

Acredito que somos despreparados para esses atendimentos e as situações intersetoriais são insuficientes e costumam demandar tempo, sendo um desgaste para nós profissionais diante das recusas de assistência dos demais setores. $(\mathrm{P} 4)$

Temos muitos[sic] que discutir coletivamente e melhorar enquanto diálogo entre a rede de serviços. (P11)

[...] conhecer a rede de serviços, identificar casos suspeitos e direcionar o atendimento. (P13)

Os profissionais da enfermagem são vistos, muitas vezes, pela equipe, como responsáveis pelo acolhimento e notificação dos casos de violência.

Ainda falta sensibilizar ainda mais todas as categorias, não só a enfermagem, mas médicos, enfim [...] todas as categorias da área da saúde, agente de saúde que é o que vai na casa, que às vezes pode estar presenciando [...]. (P1)

[...] eu acho que até o pessoal da enfermagem mesmo, sempre orienta eles, já comunica se está tendo alguma coisa. (P14)

Os profissionais mencionam a necessidade de sensibilizar os profissionais de toda a equipe sobre os outros pontos da rede de assistência, com dispositivos que podem atuar no adequado acolhimento às mulheres. A enfermagem é vista como uma das profissões responsáveis pelo acolhimento em situações de violência doméstica.

Contexto da pandemia da Covid-19: [...] aumentou [a violência] pela situação de estresse e maior tempo juntos em casa

Os participantes entrevistados no segundo momento da pesquisa, quando questionados sobre a ocorrência de mudanças no cenário da violência doméstica no contexto da pandemia da 
Covid-19, relataram em sua percepção que ocorreu um aumento do número de casos. Também, foi consenso, para quem respondeu com afirmativa e justificou sua opinião, que esse aumento se deu devido ao maior tempo de convivência com o agressor, em decorrência da medida de isolamento social para a contenção da pandemia, conforme os depoimentos.

[o aumento dos casos de violência doméstica contra a mulher vem acontecendo] [...] devido à convivência em tempo integral è à pressão que todos já estamos enfrentando com os medos de uma doença sem um tratamento especifico e todas as consequências ocorridas. (P15)

Aumentou pela situação de estresse e maior tempo juntos em casa.(P12)

Aumento de casos verificados pelos noticiários, levando em conta a situação de isolamento com as consequências econômicas e emocionais que o acompanham. (P13)

Os usuários estão mais distantes dos serviços, mudanças econômicas e de rotina de vida podem ter influência nisso. (P17)

Para as participantes, as diversas situações de estresse desencadeadas durante a pandemia podem contribuir para o aumento da violência doméstica. Também, o isolamento social e as mudanças ocorridas na rotina e renda das famílias podem levar aos conflitos intrafamiliares.

\section{Discussão}

A atenção básica pode ser o local de acesso de primeiro contato, longitudinal e de atendimento integral às pessoas e atuar na identificação, em sua área de abrangência, das mulheres vítimas de violência doméstica. ${ }^{2}$ Portanto, a unidade de saúde deve estar apta a oferecer o suporte necessário às vítimas, colaborando para que possam decidir com clareza sobre o enfrentamento da situação. ${ }^{14}$ No contexto pandêmico, o mapeamento das situações de violência no território vivo torna-se fundamental para a prevenção dos casos, acolhimento das vítimas e encaminhamentos assistenciais pela rede de serviços. ${ }^{15}$

Os profissionais entrevistados sabem identificar o que é violência doméstica. Estes entendem que, para além dos aspectos físicos e mentais da violência, existem a violência verbal e a econômica. Mas, muitas vezes, apesar de reconhecerem a violência, os participantes se sentem inseguros e precisam desenvolver habilidades interpessoais para abordar o assunto da violência doméstica com mulheres. ${ }^{16} \mathrm{O}$ reconhecimento do que é violência é importante, mas o que se torna 
fundamental para a atuação na atenção básica é o que a equipe fará para o acolhimento das mulheres que sofrem violência e quais serão os dispositivos da rede de serviços que serão necessários para atender às mulheres adequadamente. ${ }^{2}$

Situações complexas podem apresentar-se nas unidades de saúde, exigindo da equipe de saúde encaminhamentos para diferentes setores. A violência doméstica exige que os profissionais extrapolem as ações ofertadas apenas pelas organizações de saúde, buscando os serviços que possam cuidar de modo integral e que estejam interligados, evitando rotas críticas. ${ }^{17}$ Pensando nisso, toda a equipe envolvida no cuidado precisa pensar as ações desde a notificação até o encaminhamento dos casos.

Além de encaminhamentos complexos, existe a necessidade de constante identificação da rede de atenção, que pode sofrer frequentes alterações. Isso exige de todos os profissionais a pactuação e construção desse caminho assistencial, uma vez que identificar a rede de proteção contra a violência é urgente para o enfrentamento do problema. ${ }^{18}$ Muitas vezes, as mulheres que decidem romper com o agressor necessitam de diferentes serviços de atendimento, que vão desde o acolhimento jurídico, social até cuidados de saúde física e mental. ${ }^{19}$ Para que esse caminho seja percorrido, é preciso um trabalho intersetorial, um diálogo entre os serviços. ${ }^{17}$

Esta articulação coloca no profissional envolvido no atendimento a responsabilidade de identificar e direcionar opções de moradia nas casas de apoio, medidas protetivas, auxílios socioeconômicos e apoio para as crianças em idade escolar, entre outros. Esse direcionamento pela rede de cuidados que a situação de violência impõe, em alguns casos, não é entendido pelos profissionais como sendo função da área da saúde. Devido a isso, acaba sendo negligenciado, fazendo com que essas mulheres construam sua caminhada assistencial sozinhas. ${ }^{19}$

Ainda, sobre o perfil dos profissionais que atuam na atenção básica, outros fatores que podem interferir na atuação vão desde a formação inadequada até a ocorrência de conflitos morais e éticos que culminam na culpabilização e responsabilização das vítimas pela situação de violência em que se encontram. ${ }^{20} \mathrm{O}$ inadequado preparo e a não identificação da situação de violência levam a 
Violência doméstica à mulher: percepção e abordagem profissional na aten... | 14

uma invisibilidade da situação e, consequentemente, à ausência de notificação no sistema de vigilância em saúde. ${ }^{21}$ Essa situação advém de uma dificuldade enfrentada pelos profissionais relacionada à escassez de regulamentos que firmem procedimentos técnicos para esse fim e pela ausência de mecanismos legais de proteção aos profissionais encarregados de notificar. ${ }^{22-23}$

O desconhecimento de alguns profissionais sobre esta temática pode ser percebido, pois em alguns depoimentos prevaleceu a visão de que a enfermagem é a responsável pelo acolhimento das mulheres na unidade de saúde. A centralidade do cuidado talvez seja em maior grau depositada na enfermagem pelo seu papel de escuta e triagem em muitas unidades de saúde. ${ }^{19}$ No entanto, na discussão sobre a formação para o Sistema Único de Saúde (SUS), o papel de cada profissional da equipe de saúde da família e da atenção básica tensiona esse paradigma. O conhecimento dos profissionais sobre outros serviços, que não os próprios, não pode ser parcial, devendo existir fluxograma estabelecido e trabalho coordenado em rede. ${ }^{17}$

Nesse sentido, várias publicações abordam a importância da inserção nos currículos da graduação de discussões que contemplem o gênero e o acolhimento. ${ }^{16-17,19} \mathrm{O}$ bom atendimento não pode depender da boa vontade dos profissionais, que, muitas vezes, não têm acesso à formação adequada nem durante suas formações acadêmicas nem em serviço. ${ }^{17}$

Para contribuir para a mudança deste cenário e diante da importante demanda, a Educação Permanente em Saúde poderia ser uma ferramenta para atualizar, qualificar e auxiliar na formação em serviço dos profissionais, tendo em vista a dinamicidade de serviços e ações jurídicas, sociais e de saúde que precisam ser acionados ao mesmo tempo na rede para assistir mulheres vítimas de violência. ${ }^{24}$ A educação permanente pode incentivar os trabalhadores capacitados a serem agentes multiplicadores do conhecimento, a fim de contribuir para a diminuição do número de casos de violência contra as mulheres com a qualificação profissional de outros trabalhadores da saúde. ${ }^{25}$

Os participantes relataram a percepção de que os casos de violência durante a pandemia da Covid-19 aumentaram. No atual cenário de isolamento social, com maior frequência as mulheres são vigiadas e impedidas de conversar com familiares e amigos, o que pode ampliar a margem de 
ação para a manipulação. ${ }^{26} \mathrm{O}$ controle das finanças domésticas também se torna mais acirrado, pela perda do controle financeiro pelo homem, devido às consequências econômicas do cenário de pandemia, e por sua presença mais próxima em um ambiente que é mais comumente dominado pelas mulheres. A perspectiva da perda de poder masculino fere diretamente a sua imagem, servindo de gatilho para comportamentos violentos. Os aspectos relacionados a estresse, medos e consequentes divergências conjugais implícitas ao contexto de instabilidade também colaboram..$^{10,27}$

A menor frequência das mulheres nos serviços de saúde também pode contribuir para a ocorrência das violências. ${ }^{22}$ Observa-se que, com a necessidade de permanecer em casa e a dificuldade de acesso à unidade de saúde, a identificação dos casos de violência e a possibilidade de a mulher falar sobre o que está ocorrendo com os profissionais foram afetadas. ${ }^{27}$

A pandemia reconfigurou o funcionamento de muitos serviços, não só na saúde, mas em outras áreas, como delegacias, órgãos de assistência social, casas de apoio, escolas, igrejas. Enfim, todos estes locais, que antes podiam ser acessados mais facilmente, com a pandemia, tiveram horários diferenciados ou até foram fechados temporariamente com a não adaptação dos serviços de modo on-line. ${ }^{22}$ Nesta perspectiva, o adequado preparo dos profissionais e ações permanentes de educação em serviço podem ter impactos positivos e produzir cuidado qualificado, pois mesmo na pandemia as reconfigurações da rede intersetorial poderiam ser apreendidas e os profissionais indicariam serviços adequadamente. ${ }^{15}$

Ao finalizar o trabalho, pode-se observar que a nossa limitação foi a ocorrência da pandemia, que ocasionou o atraso e a necessidade de reorientação da coleta dos dados. As pesquisadoras observaram que, após a pandemia, alguns profissionais foram remanejados para outros serviços, estavam adoecidos por Covid-19 ou ainda sobrecarregados em suas funções laborais, e isso pode ter influenciado na participação na pesquisa. Além disso, o instrumento on-line limitou a interação com os pesquisadores e ainda pode não ter sido entendido por todas as pessoas, devido à dificuldade em acessar e responder um instrumento digital.

$\mathrm{Na}$ área assistencial, destacam-se as contribuições para a ampliação de espaços de 
qualificação dos profissionais para atuarem na rede intersetorial, inclusive durante a pandemia. No ensino, ressalta-se a importância da incorporação e maior sensibilização dos profissionais desde a formação para trabalhar com essa temática. Sugere-se, para o serviço, a busca por técnicas de acolhimento e escuta voltadas a orientar profissionais da enfermagem, a fim de que se sintam preparados para ouvir e promover cuidado em saúde sempre que se fizer necessário. Torna-se importante desenvolver pesquisas que possam atuar na identificação da rede intersetorial, apontando quais são os dispositivos necessários para a adequada assistência às mulheres que sofrem violências. Também, instrumentalizar os profissionais da rede para práticas de pesquisa que possam mostrar as mudanças que acontecem no percurso assistencial, como durante a pandemia, pois ocorreram modificações em diversos serviços de atendimento a essas mulheres.

\section{Considerações finais}

Os profissionais da saúde identificam o que é violência doméstica e suas diferentes manifestações. Porém, muitos desconhecem a rede assistencial e os serviços de acolhimento. Este desconhecimento implica na ausência da notificação e na invisibilidade dos casos. As capacitações e a implementação de ações de educação permanente em saúde poderiam atuar favoravelmente na constante qualificação da equipe de saúde para atuar na atenção básica.

No período da pandemia, os profissionais observaram, em suas práticas, um acréscimo dos casos devido ao isolamento social e ao aumento de situações estressoras. É apontada pelos participantes a necessidade de conhecimento da rede de serviços e do aumento de ações intersetoriais, devido à dinamicidade da assistência às mulheres em violência doméstica. 


\section{Referências}

1. BRASIL. Ministério da Saúde. Portaria no 2.436, de 21 de setembro de 2017. Aprova a Política Nacional de Atenção Básica, estabelecendo a revisão de diretrizes para organização da Atenção Básica, no âmbito do Sistema Único de Saúde (SUS). Diário Oficial da União: seção 1, Brasília, DF, n. 183, p. 68, 22 set. 2017.

2. Esperandio EG, Moura ATMS, Favoreto CAO. Violência íntima: experiências de mulheres na Atenção Primária à Saúde no Rio de Janeiro, RJ, Brasil. Interface (Botucatu). 2020;24(Supl 1): e190707. doi: https://doi.org/10.1590/Interface.190707

3. Organização Panamericana de Saúde, Organização Mundial da Saúde (OPAS/OMS Brasil). - Violência contra as mulheres [Internet]. Brasília (DF): OPAS/OMS Brasil; 2017 [cited 2021 Feb 05]. Available from: https://www.paho.org/pt/topics/violence-against-women

4. Ministério da Saúde (BR). Política Nacional de Enfrentamento à Violência Contra as Mulheres [Internet]. Brasília (DF): Ministério da Saúde; 2011 [acesso em 2011 set 24]. Disponível em: https:/www12.senado.leg.br/institucional/omv/acoes-contra-violencia/politica-nacional-de-enfrentamento-aviolencia-contra-as-mulheres

5. Conselho Nacional de Justiça (BR). Violência contra a Mulher: XIII Jornada: Lei Maria da Penha [Internet]. 2016 [acesso em 10 jan 2021]. Brasília (DF): Conselho Nacional de Justiça; 2021 [acesso em 2021 jan 10]. Disponível em: https://www.cnj.jus.br/programas-e-acoes/violencia-contra-a-mulher/

6. Centro de Informática e Automação do Estado de Santa Catarina (CIASC). SISP - Sistema Integrado de Segurança Pública [Internet]. Florianópolis: CIASC; 2019 [acesso em 2020 jun 10]. Disponível em: https://www.ciasc.sc.gov.br/produto/sisp-sistema-integrado-de-seguranca-publica/

7. Fórum Brasileiro de Segurança Pública. Anuário Brasileiro de Segurança Pública 2021 [Internet]. 2021 [acesso em 2021 jul 21]. Disponível em: https:/forumseguranca.org.br/wp-content/uploads/2021/07/anuario-2021completo-v6-bx.pdf

8. ONU Mulheres Brasil. Violência contra mulheres e meninas é pandemia invisível, afirma diretora executiva da ONU Mulheres [Internet]. Brasília (DF): Onu Mulheres Brasil; 2020 [acesso em 2021 jul 21]. Disponível em: https://www.onumulheres.org.br/noticias/violencia-contra-as-mulheres-e-meninas-e-pandemia-invisivelafirma-diretora-executiva-da-onu-mulheres/

9. Barbosa JPM, Lima RCD, Santos GBM, Lanna SD, Andrade MAC. Interseccionalidade e violência contra as mulheres em tempos de pandemia de covid-19: diálogos e possibilidades. Saúde Soc. 2021;30(2):e200367. doi: https://doi.org/10.1590/S0104-12902021200367

10. Vieira PR, Garcia LP, Maciel ELN. Isolamento social e o aumento da violência doméstica: o que isso nos revela? Rev Bras Epidemiol. 2020;23:e200033. doi: https://doi.org/10.1590/1980-549720200033

11. Rodrigues EAS, Tavares R, Melo VH, Silva JM, Melo EM. Violência e atenção primária à saúde: percepções e vivências de profissionais e usuários. Saúde Debate. 2018;42(Spec No 4):55-66. doi: https://doi.org/10.1590/0103$11042018 S 404$

12. Campos B, Tchalekian B, Paiva V. Violência contra a mulher: vulnerabilidade programática em tempos de 
Violência doméstica à mulher: percepção e abordagem profissional na aten... | 18

SARS-CoV-2/Covid-19 em São Paulo. Psicol Soc. 2020;32:e020015. doi: https://doi.org/10.1590/18070310/2020v32240336

13. Bardin L. Análise de conteúdo. São Paulo: Edições 70; 2011.

14. Lima JCV, Santos RC, Silva JC, Silva RDSC, Souto CMRM, Souto RQ, et al. Rastreio e encaminhamento de casos de violência contra a mulher por enfermeiras na estratégia de saúde da família. Cogitare Enferm. 2020;25:e65579. doi: http://dx.doi.org/10.5380/ce.v25i0.65579

15. Cortes LF, Arboit J, Gehlen RGS, Tassinari TT, Vieira LB, Padoin SMM, et al. Desafios na proteção às mulheres em situação de violência no contexto de pandemia da COVID-19. Ciênc Cuid Saúde. 2020;19:e27984. doi: https://doi.org/10.4025/cienccuidsaude.v19i0.54847

16. Vargas EB, Santos EH. Atuação do profissional de saúde na atenção primária frente à violência doméstica à mulher [monografia]. Palhoça: Universidade do Sul de Santa Catarina; 2017 [acesso em 2021 fev 05 fev]. Disponível em: https://repositorio.animaeducacao.com.br/handle/ANIMA/4881

17. Vieira EM, Hasse M. Percepções dos profissionais de uma rede intersetorial sobre o atendimento a mulheres em situação de violência. Interface (Botucatu). 2017;21(60):51-62. doi: https://doi.org/10.1590/180757622015.0357

18. Souza TMC, Rezende FF. Violência contra mulher: concepções e práticas de profissionais de serviços públicos. Estud Interdiscip Psicol [Internet]. 2018 [acesso em 2021 abr 10];9(2):21-38. Disponível em: http://pepsic.bvsalud.org/scielo.php?script=sci_arttext\&pid=S2236-64072018000200003\&lng=pt\&nrm=iso

19. Oliveira TM, Ferigato SH. A atenção às mulheres vítimas de violência doméstica e familiar: a construção de tecnologias de cuidado da terapia ocupacional na atenção básica em saúde. Cad Bras Ter Ocup. 2019;27(3):50821. doi: https://doi.org/10.4322/2526-8910.ctoao1729

20. Souza AAC, Cintra RB. Conflitos éticos e limitações do atendimento médico à mulher vítima de violência de gênero. Rev Bioét. 2018;26(1):77-86. doi: https://doi.org/10.1590/1983-80422018261228

21. Viana AL, Lira MOSC, Vieira MCA, Sarmento SS, Souza APL. Violência contra a mulher. Rev Enferm UFPE On Line. 2018;12(4):923-9. doi: https://doi.org/10.5205/1981-8963-v12i4a110273p923-929-2018

22. Mendonça CS, Machado DF, Almeida MAS, Castanheira ERL. Violência na Atenção Primária em Saúde no Brasil: uma revisão integrativa da literatura. Ciênc Saúde Colet. 2020;25(6):2247-57. doi: http://dx.doi.org/10.1590/1413-81232020256.19332018

23. Oliveira BG, Freire IV, Assis CS, Sena LS, Boery RNSO, Yarid SD. Responsabilidade dos profissionais de saúde na notificação dos casos de violência. Rev Bioét. 2018;26(3):403-11. doi: https://doi.org/10.1590/198380422018263260

24. Secretaria de Estado da Saúde (SC). Protocolo de atenção às vítimas de violência sexual dos municípios da região da AMOSC [Internet]. Chapecó (SC): Secretaria de Estado da Saúde do Estado de Santa Catarina; 2015 [acesso em 2021 mar 02]. Disponível em: https://www.saude.sc.gov.br/index.php/documentos/atencaobasica/atencao-as-pessoas-em-situcao-de-violencia/eventos/seminario-formacao-de-rede-intersetorial/10534protocolo-regional/file

25. Santos JPB, Lima RRT. Educação permanente em saúde para qualificar o acolhimento às mulheres vítimas 
19 | Odorcik B, Ferraz BP, Bastos KC, Rossetto M

de violência: debatendo uma proposta. Res Soc Dev. 2020;9(1):e173911859. doi: http://dx.doi.org/10.33448/rsdv9i1.1859

26. Campos B, Tchalekian B, Paiva V. Violência contra a mulher: vulnerabilidade programática em tempos de Sars-CoV-2/ COVID-19 em São Paulo. Psicol Soc. 2020 Sep 04;32:e020015. doi: http://dx.doi.org/10.1590/18070310/2020v32240336

27. Roesch E, Amin A, Gupta J, García-Moreno C. Violence against women during Covid-19 pandemic restrictions. BMJ. 2020 may 07;369:m1712. doi: https://doi.org/10.1136/bmj.m1712

Editora Científica Chefe: Cristiane Cardoso de Paula

Editora Associada: Graciela Dutra Sehnem

\section{Autor correspondente}

Maíra Rossetto

E-mail: maira.rossetto@uffs.edu.br

Endereço: Travessa Tijucas 109e, apt 802, bairro Presidente Médice, Chapecó, SC. CEP: 89801307

\section{Contribuições de Autoria}

\section{1 - Bruna Odorcik}

Concepção ou desenho do estudo/pesquisa, análise e/ou interpretação dos dados, revisão final com participação crítica e intelectual no manuscrito.

\section{2 - Brígida da Penha Ferraz}

Análise e/ou interpretação dos dados, revisão final com participação crítica e intelectual no manuscrito

\section{3 - Karina Castilhos Bastos}

Análise e/ou interpretação dos dados, revisão final com participação crítica e intelectual no manuscrito

\section{4 - Maíra Rossetto}

Concepção ou desenho do estudo/pesquisa, análise e/ou interpretação dos dados, revisão final com participação crítica e intelectual no manuscrito.

\section{Como citar este artigo}

Odorcik B, Ferraz BP, Bastos KC, Rossetto M. Violência doméstica à mulher: percepção e abordagem profissional na atenção básica na pandemia de Covid-19. UFSM. 2021 [Acesso em: Anos Mês Dia]; vol.11 e74: 1-19. DOI: https://doi.org/10.5902/2179769265865 